\title{
Phase-dependent interaction in a four-level atomic configuration
}

\author{
Giovanna Morigi, ${ }^{1, *}$ Sonja Franke-Arnold, ${ }^{2}$ and Gian-Luca $\mathrm{Oppo}^{2}$ \\ ${ }^{1}$ Max-Planck-Institut für Quantenoptik, Hans-Kopfermannstrasse 1, D-85748 Garching, Germany \\ ${ }^{2}$ Department of Physics, University of Strathclyde, 107 Rottenrow, Glasgow G4 ONG, United Kingdom
}

(Received 21 June 2002; published 18 November 2002)

\begin{abstract}
We study a four-level atomic scheme interacting with four lasers in a closed-loop configuration with a $\diamond$ (diamond) geometry. We investigate the influence of the laser phases on the steady state. We show that, depending on the phases and the decay characteristic, the system can exhibit a variety of behaviors, including population inversion and complete depletion of an atomic state. We explain the phenomena in terms of multiphoton interference, and compare our results with the phase-dependent phenomena in the double- $\Lambda$ scheme. This investigation may be useful for developing nonlinear optical devices, and for the spectroscopy and laser cooling of alkali-earth atoms.
\end{abstract}

DOI: 10.1103/PhysRevA.66.053409

PACS number(s): 42.50.Md, 32.80.Qk

\section{INTRODUCTION}

Absorption and emission of monochromatic light in twolevel atomic transitions are well-understood processes in quantum optics [1]. Their properties, however, can change drastically if transitions to a third atomic level have to be included. This is the case, for instance, in the $\Lambda$ configuration where two stable states are coupled to a common excited state by laser fields, thus providing two excitation paths which can interfere. This interference lies at the heart of coherent population trapping (CPT) [2]. Here, destructive interference between the transition amplitudes gives rise to a superposition of atomic states (dark state) that is decoupled from coherent radiation but populated by spontaneous emission. Consequently the atom becomes "trapped" in this coherent superposition.

For configurations like the $\Lambda$ scheme, the relative phase of the laser fields does not affect the steady-state dynamics, in the sense that there always exists a reference frame in which the Rabi frequencies are real. This is no longer fulfilled in closed-loop configurations [3,4], i.e., when a set of atomic states is (quasi) resonantly coupled by laser fields, such that each state of the set is connected to any other via two different paths of coherent photon scattering: In this case, the relative phase $\Phi$ between the transitions determines the interference and hence critically influences the dynamics and the steady state of the system [3-5]. Previous studies of closed-loop configurations often featured double- $\Lambda$ systems, where two-stable or metastable states are, each, coupled to two common excited states [5-8]. These works have shown a rich variety of nonlinear optical phenomena.

In this paper, we investigate the phase-dependent dynamics of a closed-loop configuration, consisting of four transitions driven by lasers. One ground state is coupled in a $V$-type structure to two intermediate states, which are themselves coupled to a common excited state in a $\Lambda$-type structure. We label this system the $\diamond$ (diamond) scheme. The steady state of the $\diamond$ scheme, like that of the double- $\Lambda$

\footnotetext{
*Present address: Abteilung Quantenphysik, University of Ulm, Albert-Einstein-Allee 11, D-89081 Ulm, Germany.
}

configuration, is a periodic function of the relative phase $\Phi$ between the excitation paths, which contribute to the scattering between any initial and final state of the scheme. In particular, the steady state is determined by the concurrence of the phase-dependent Hamiltonian dynamics and the relaxation processes. We will show that, depending on $\Phi$ and on the lifetimes of the intermediate states, the $\diamond$ system can show a variety of behaviors including population inversion, CPT, and phase-dependent refractive indices. It is worth noting that the double- $\Lambda$ and the $\diamond$ schemes are governed by the same Hamiltonian, but are characterized by different relaxation processes. This results in critical differences in the dynamics, which we will point out in our discussion.

Excitation configurations like the $\diamond$ scheme have been investigated in the literature as a model for observing pressure-induced resonances [9], and can be found, for instance, in experiments with gases of alkali-earth atoms which aim at optical frequency standards [10] or at reaching the quantum degeneracy regime by all-optical means [11]. Our investigation may contribute to the spectroscopy of these systems and to the development of new and efficient methods of laser cooling.

This paper is organized as follows. In Sec. II we introduce the model and the basic equations. We discuss the properties of the system, and identify some relevant parameter regimes. In Sec. III we calculate the steady-state solutions as a function of the relative phase between excitation paths for certain parameters, and discuss the results. In Sec. IV we draw the conclusions and present some outlooks.

\section{THE MODEL}

We consider a dilute gas of atoms of mass $m$, in a thermal distribution at temperature $T$. The atoms are free, and interact with a multichromatic light field. For a sufficiently dilute gas, each atom interacts individually with the light, which couples to a set of atomic levels as depicted in Fig. 1. This set contains a ground state $|1\rangle$, two intermediate states $|2\rangle$ and $|3\rangle$, and an excited state $|4\rangle$. The transitions $|1\rangle$ $\rightarrow|2\rangle,|3\rangle$ and $|2\rangle,|3\rangle \rightarrow|4\rangle$ are optical dipoles with decay rates $\gamma_{2}, \gamma_{3}, \gamma_{42}$, and $\gamma_{43}$, respectively. Each dipole transition is driven resonantly by a laser, which is here consid- 


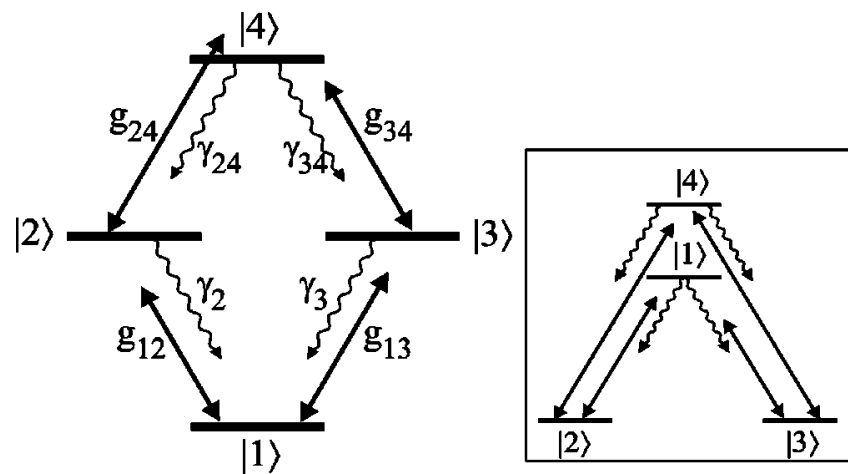

FIG. 1. Atomic level scheme and nomenclature or the relevant levels for the $\diamond$ configuration. The inset displays the double- $\Lambda$ system for comparison.

ered to be a classical running wave, propagating along the $\hat{z}$ axis. The laser coupling to the transition $|i\rangle \rightarrow|j\rangle$ is characterized by the frequency $\omega_{i j}$ and the wave vector $k_{i j}$, while the strength of the coupling is given by the Rabi frequency $g_{i j} \mathrm{e}^{\mathrm{i} \chi_{i j}}$, where $g_{i j}$ is real and $\chi_{i j}$ is a constant phase, determined by the phase of the atomic dipole and by the phase of the laser at time $t=0$ and position $z=0$. The state of one atom at time $t$ is described by the density matrix $\sigma$, which obeys the master equation

$$
\frac{\partial}{\partial t} \sigma=\frac{1}{\mathrm{i} \hbar}[H(t), \sigma]+\mathcal{L} \sigma .
$$

Here, the Hamiltonian $H(t)$ contains the coherent dynamics of the atom and the Liouvillian $\mathcal{L}$ describes the relaxation processes. The Hamiltonian $H(t)$ reads

$$
\begin{aligned}
H(t)= & \frac{p_{z}^{2}}{2 m}+\sum_{j=2}^{4} \hbar \omega_{j}|j\rangle\langle j| \\
& +\frac{\hbar}{2} \sum_{j=2,3}\left(g_{1 j} \mathrm{e}^{-i\left(\omega_{1 j} t-k_{1 j} z+\chi_{1 j}\right)}|j\rangle\langle 1|\right. \\
& \left.+g_{j 4} \mathrm{e}^{-i\left(\omega_{j 4} t-k_{j 4} z+\chi_{j 4}\right)}|4\rangle\langle j|+\text { H.c. }\right) .
\end{aligned}
$$

The first term corresponds to the kinetic energy of the atomic center of mass, where $p_{z}$ is its momentum along the $\hat{z}$ axis (for simplicity, we consider only the component of the atomic motion along the $\hat{z}$ axis). The second term corresponds to the internal Hamiltonian, where $\hbar \omega_{j}$ denote the energies of the atomic states $|j\rangle$ relative to the energy of the state $|1\rangle$. The remaining term describes the atom-laser interaction.

The relaxation processes are assumed to be solely radiative, and the Liouvillian $\mathcal{L}$ in (1) has the form:

$$
\begin{aligned}
\mathcal{L} \sigma= & \sum_{j=2,3} \gamma_{4 j}|j\rangle\langle 4|\sigma| 4\rangle\langle j|-\frac{\gamma_{4}}{2}(|4\rangle\langle 4|\sigma+\sigma| 4\rangle\langle 4|) \\
& +\sum_{j=2,3} \gamma_{j}\left[|1\rangle\langle j|\sigma| j\rangle\langle 1|-\frac{1}{2}(|j\rangle\langle j|\sigma+\sigma| j\rangle\langle j|)\right],
\end{aligned}
$$

where $\gamma_{4}=\gamma_{42}+\gamma_{43}$ is the total decay rate from level $|4\rangle$. In writing Eq. (3) we have neglected the mechanical effects of light on the atomic center-of-mass motion that accompany the photon emission. In fact, in the main body of the paper we assume that the thermal energy $k_{B} T$ is much larger than the recoil energies $\hbar^{2} k_{i j}^{2} / 2 m$. In this limit, we can treat the atomic motion classically and neglect the effects of the photon recoil on the center-of-mass dynamics [12].

The Hamiltonian (1) is explicitly time dependent. For the configuration we consider here there exists in general no reference frame in which this explicit time dependence can be eliminated. This is a characteristic of "closed-loop" configurations [4], and it is a manifestation of the intrinsic phase sensitivity of the dynamics. However, in an adequate reference frame, the Rabi frequencies can be chosen such that only one is complex, with its phase $\Phi$ being a function of all laser phases. Without loss of generality, we move to a reference frame where $\Phi$ is associated with the laser coupling to the transition $|3\rangle \rightarrow|4\rangle$, so that the coherent dynamics of the system is now described by the Hamiltonian [3]

$$
\begin{aligned}
\tilde{H}(\Phi)= & \frac{p_{z}^{2}}{2 m}+\sum_{j=2}^{4} \hbar \delta_{j}|j\rangle\langle j|+\frac{\hbar}{2}\left(g_{12}|2\rangle\left\langle 1\left|+g_{13}\right| 3\right\rangle\langle 1|\right. \\
& \left.+g_{24}|4\rangle\left\langle 2\left|+g_{34} \mathrm{e}^{\mathrm{i} \Phi}\right| 4\right\rangle\langle 3|+\text { H.c. }\right),
\end{aligned}
$$

where $\Phi=\Phi(t, z)$. The detunings $\delta_{j}$ in Eq. (4) are given by

$$
\begin{gathered}
\delta_{2}=\left(\omega_{2}-\omega_{12}\right)+\frac{k_{12} p_{z}}{m}+\frac{\hbar k_{12}^{2}}{2 m}, \\
\delta_{3}=\left(\omega_{3}-\omega_{13}\right)+\frac{k_{13} p_{z}}{m}+\frac{\hbar k_{13}^{2}}{2 m}, \\
\delta_{4}=\left(\omega_{4}-\omega_{12}-\omega_{24}\right)+\frac{\left(k_{12}+k_{24}\right) p_{z}}{m}+\frac{\hbar\left(k_{12}+k_{24}\right)^{2}}{2 m},
\end{gathered}
$$

and the phase $\Phi$ is defined as

$$
\Phi=\Delta \omega t-\Delta k z+\Delta \chi,
$$

where

$$
\begin{gathered}
\Delta \omega \doteq \omega_{12}+\omega_{24}-\omega_{13}-\omega_{34}, \\
\Delta k \doteq k_{12}+k_{24}-k_{13}-k_{34}, \\
\Delta \chi \doteq \chi_{12}+\chi_{24}-\chi_{13}-\chi_{34} .
\end{gathered}
$$

The phase $\Phi$ is the relative phase between the two excitation paths characterizing any transition between two atomic states. This phase is in general time and position dependent, and it results from the multiphoton detuning $\Delta \omega$, the wavevector mismatch $\Delta k$, and the initial laser and atomic-dipole phases, $\Delta \chi$.

We denote with $\rho$ the density matrix in the new reference frame. Its evolution is described by the master equation

$$
\frac{\partial}{\partial t} \rho=\frac{1}{\mathrm{i} \hbar}[\tilde{H}(\Phi), \rho]+\mathcal{L} \rho .
$$


The one-atom density matrix is given by $\rho$ $=\int \mathrm{d} z \mathrm{~d} p_{z} n\left(z, p_{z}\right) \rho\left(p_{z}, z\right)$, where $n\left(z, p_{z}\right)$ is the atomic density as a function of the position and the momentum, and $\rho\left(z, p_{z}\right)$ obeys the master equation (9) at the parameters $z, p_{z}$. Denoting with $\rho_{i j}=\left\langle i\left|\rho\left(z, p_{z}\right)\right| j\right\rangle$ its elements, with $i, j=1,2,3,4$ [we drop the explicit dependence on the parameters $\left.\left(z, p_{z}\right)\right]$, the optical Bloch equations (OBE) have the form

$$
\begin{gathered}
\dot{\rho}_{11}=i \frac{g_{12}}{2}\left(\rho_{12}-\rho_{21}\right)+i \frac{g}{2}\left(\rho_{13}-\rho_{31}\right)+\gamma_{2} \rho_{22}+\gamma_{3} \rho_{33}, \\
\dot{\rho}_{22}=-i \frac{g_{12}}{2}\left(\rho_{12}-\rho_{21}\right)+i \frac{g_{24}}{2}\left(\rho_{24}-\rho_{42}\right)+\gamma_{42} \rho_{44}-\gamma_{2} \rho_{22}, \\
\dot{\rho}_{33}=-i \frac{g}{2}\left(\rho_{13}-\rho_{31}\right)+i \frac{g}{2}\left(\mathrm{e}^{\mathrm{i} \Phi} \rho_{34}-\mathrm{e}^{-\mathrm{i} \Phi} \rho_{43}\right)+\gamma_{43} \rho_{44} \\
-\gamma_{3} \rho_{33}, \\
\dot{\rho}_{12}=\left(\mathrm{i} \delta_{2}-\frac{\gamma_{2}}{2}\right) \rho_{12}-\mathrm{i} \frac{g_{12}}{2}\left(\rho_{22}-\rho_{11}\right)-\mathrm{i} \frac{g_{13}}{2} \rho_{32}+\mathrm{i} \frac{g_{24}}{2} \rho_{14},
\end{gathered}
$$$$
\dot{\rho}_{13}=\left(\mathrm{i} \delta_{3}-\frac{\gamma_{3}}{2}\right) \rho_{13}-\mathrm{i} \frac{g_{13}}{2}\left(\rho_{33}-\rho_{11}\right)-\mathrm{i} \frac{g_{12}}{2} \rho_{23}+\mathrm{i} \frac{g_{34}}{2} \mathrm{e}^{\mathrm{i} \Phi} \rho_{14},
$$$$
\dot{\rho}_{24}=\left(-\mathrm{i}\left(\delta_{2}-\delta_{4}\right)-\frac{\gamma_{2}+\gamma_{4}}{2}\right) \rho_{24}-\mathrm{i} \frac{g_{24}}{2}\left(\rho_{44}-\rho_{22}\right)-\mathrm{i} \frac{g_{12}}{2} \rho_{14}
$$$$
+\mathrm{i} \frac{g_{34}}{2} \mathrm{e}^{-\mathrm{i} \Phi} \rho_{23},
$$$$
\dot{\rho}_{34}=\left(-\mathrm{i}\left(\delta_{3}-\delta_{4}\right)-\frac{\gamma_{3}+\gamma_{4}}{2}\right) \rho_{34}-\mathrm{i} \frac{g_{34}}{2} \mathrm{e}^{-\mathrm{i} \Phi}\left(\rho_{44}-\rho_{33}\right)
$$$$
-\mathrm{i} \frac{g_{13}}{2} \rho_{14}+\mathrm{i} \frac{g_{24}}{2} \rho_{32},
$$$$
\dot{\rho}_{14}=\left[\mathrm{i} \delta_{4}-\frac{\gamma_{4}}{2}\right] \rho_{14}-\mathrm{i} \frac{g_{12}}{2} \rho_{24}-\mathrm{i} \frac{g_{13}}{2} \rho_{34}+\mathrm{i} \frac{g_{24}}{2} \rho_{12}
$$$$
+\mathrm{i} \frac{g_{34}}{2} \mathrm{e}^{-\mathrm{i} \Phi} \rho_{13},
$$$$
\dot{\rho}_{23}=\left[-\mathrm{i}\left(\delta_{2}-\delta_{3}\right)-\frac{\gamma_{2}+\gamma_{3}}{2}\right] \rho_{23}-\mathrm{i} \frac{g_{12}}{2} \rho_{13}-\mathrm{i} \frac{g_{24}}{2} \rho_{43}
$$$$
+\mathrm{i} \frac{g_{13}}{2} \rho_{21}+\mathrm{i} \frac{g_{34}}{2} \mathrm{e}^{\mathrm{i} \Phi} \rho_{24},
$$

and $\rho_{j i}=\left(\rho_{i j}\right) *$. In the following we will refer to the diagonal elements as "populations," giving the occupation of the atomic states, and to the off-diagonal elements as "one- photon coherences" or "two-photon coherences," depending on whether the involved states are coupled at lowest order by the scattering of one or two photons, respectively.

\section{A. Discussion}

Equations (10)-(19) exhibit a parametric time dependence, which enters through the phase $\Phi$ as in Eq. (5). Such behavior imposes limitations on the existence of a steadystate solution. Neglecting the coupling of the internal degrees of freedom with the external ones, two cases can be identified where the internal steady state exists: (i) when the scheme is driven well below saturation, and/or (ii) for $\Delta \omega$ $=0$. In (i), the processes leading to the absorption of two photons are negligible, and the relevant dynamics takes place between the ground state $|1\rangle$ and the intermediate states $|2\rangle,|3\rangle$, coupled in a (open-loop) $V$-type configuration. In (ii), the steady-state solution is defined for any value of the other parameters. For $\Delta \omega=0$ and $\Delta k=0, \Phi$ is determined solely by the initial laser and dipole phases. While the dipole phases are fixed by the quantum numbers of the atomic transitions, the laser phases can be modified. In this regime, which we study in the remainder of the paper, the dynamics of the $\diamond$ configuration shares some analogies with an interferometer, the arms of which are formed by the multiphoton excitation paths and the phase difference between them is given by the relative phase $\Phi$. Such analogy was first drawn in Ref. [3] for the coherent dynamics of a closed-loop scheme. In this spirit we interpret some of the results, presented below and in the following section, which have been obtained in the presence of relaxation processes.

An interesting manifestation of the phase-dependent dynamics is the probability of two-photon absorption on the transition $|1\rangle \rightarrow|4\rangle$. In the limit of weak excitations it has the form $\quad P_{1 \rightarrow 4} \propto \mid g_{12} g_{24} /\left(\delta_{2}-\mathrm{i} \gamma_{2} / 2\right)+g_{13} g_{34} \exp (\mathrm{i} \Phi) /\left(\delta_{3}\right.$ $\left.-\mathrm{i} \gamma_{3} / 2\right)\left.\right|^{2}$, where the first and second terms on the righthand side describe the transitions via the intermediate states $|2\rangle$ and $|3\rangle$, respectively. Thus, both the phase difference $\Phi$ as well as the ratio of the laser detunings $\delta_{2}, \delta_{3}$ and the decay rates $\gamma_{2}, \gamma_{3}$ determine the interference between the two paths. In the special case of equal Rabi frequencies $g_{i j}$ $=g$, and for $\delta_{2}=\delta_{3}, \gamma_{2}=\gamma_{3}$, the role of $\Phi$ is singled out,

$$
P_{1 \rightarrow 4} \propto \cos ^{2} \frac{\Phi}{2} .
$$

Thus, the transition probability from the ground to the excited level is modulated by $\Phi$. In particular, it is maximal for the values $\Phi=2 n \pi$ (where $n$ is an integer), while it vanishes for $\Phi=(2 n+1) \pi$. At the latter value, no transition to $|4\rangle$ occurs. In the following section we will show that $P_{1 \rightarrow 4}$ always vanishes for $\Phi=(2 n+1) \pi$ at steady state, even when the system is driven at saturation. We remark that the appearance of this behavior requires a "symmetric" excitation configuration, meaning that each two-photon excitation path from $|1\rangle$ to $|4\rangle$ has, separately, the same probability.

A further understanding of the problem can be gained by moving to a suitable basis, following the analysis of Ref. [3]. This basis is chosen appropriate to the structure of the 

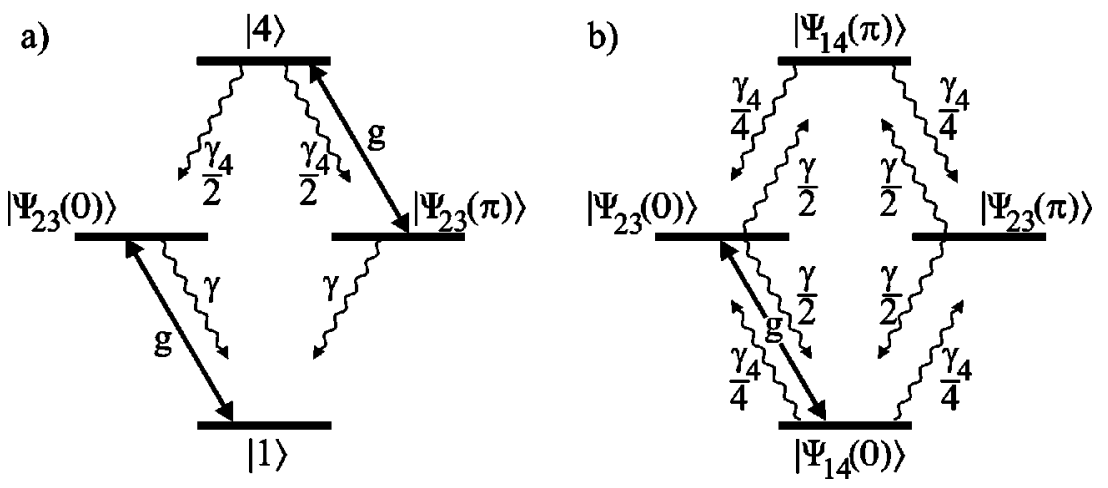

FIG. 2. Atomic level scheme in the basis of the states $\left\{|1\rangle,|4\rangle,\left|\Psi_{23}(0)\right\rangle,\left|\Psi_{23}(\pi)\right\rangle\right\}$ for the cases $\Phi=(2 n+1) \pi$ (a) and $\Phi=2 n \pi(\mathrm{b})$.

Hamiltonian (4) and the relaxation processes in Eq. (3). For $g_{i j}=g$ and $\delta_{j}=0$ the dynamics offers simple interpretations for $\Phi=n \pi$.

We first focus on the values $\Phi=(2 n+1) \pi$. Here, it is convenient to use the orthogonal basis set $\quad\left\{|1\rangle,|4\rangle,\left|\Psi_{23}(0)\right\rangle, \quad\left|\Psi_{23}(\pi)\right\rangle\right\}, \quad$ where $\left|\Psi_{23}(\theta)\right\rangle=\left[|2\rangle+\mathrm{e}^{-\mathrm{i} \theta}|3\rangle\right] / \sqrt{2}$ with $\theta=0, \pi$. In this basis, the Hamiltonian (4) can be rewritten as

$$
\widetilde{H}((2 n+1) \pi)=\frac{\hbar g}{\sqrt{2}}\left[\left|\Psi_{23}(0)\right\rangle\langle 1|+| 4\rangle\left\langle\Psi_{23}(\pi)\right|+\text { H.c. }\right] \text {, }
$$

where we have omitted the atomic motion. Thus, Eq. (21) describes two-level dynamics within the orthogonal subspaces $\left\{|1\rangle,\left|\Psi_{23}(0)\right\rangle\right\}$ and $\left\{|4\rangle,\left|\Psi_{23}(\pi)\right\rangle\right\}$ which are coupled by spontaneous decay. The coupling between states due to coherent and incoherent processes is represented in Fig. 2(a). From the structure of the decay it is evident that the atom is eventually pumped into $\left\{|1\rangle,\left|\Psi_{23}(0)\right\rangle\right\}$. Hence, the steady state of the system for this value of the phase corresponds to that of the driven two-level transition $|1\rangle \rightarrow\left|\Psi_{23}(0)\right\rangle$.

For $\Phi=2 n \pi$ we describe the system in the orthogonal basis set $\left\{\left|\Psi_{14}(0)\right\rangle,\left|\Psi_{14}(\pi)\right\rangle,\left|\Psi_{23}(0)\right\rangle,\left|\Psi_{23}(\pi)\right\rangle\right\}$, where $\left|\Psi_{14}(\theta)\right\rangle=\left[|1\rangle+\mathrm{e}^{\mathrm{i} \theta}|4\rangle\right] / \sqrt{2}$ with $\theta=0, \pi$. In this basis, the Hamiltonian $\widetilde{H}$ can be written as

$$
\widetilde{H}(2 n \pi)=\hbar g\left[\left|\Psi_{23}(0)\right\rangle\left\langle\Psi_{14}(0)\right|+\text { H.c. }\right],
$$

describing coherent two-level dynamics between the states $\left|\Psi_{23}(0)\right\rangle$ and $\left|\Psi_{14}(0)\right\rangle$. The states $\left|\Psi_{14}(\pi)\right\rangle$ and $\left|\Psi_{23}(\pi)\right\rangle$ are decoupled from the coherent drive because of destructive interference between the corresponding excitation paths, and from this point of view they are dark states. However, they are not stable, but decay with rates $\gamma_{4}$ and $\gamma_{2}+\gamma_{3}$, respectively. The level scheme in the new basis is plotted in Fig. 2(b). Here, it is evident that the system is incoherently pumped among the driven transition $\left|\Psi_{23}(0)\right\rangle \rightarrow\left|\Psi_{14}(0)\right\rangle$ and the two dark states. One could say that the steady state is determined by the competition between the Hamiltonian dynamics and the relaxation processes. Thus, some localization in one of the dark superpositions (CPT) can occur, if this is more stable than the other, i.e., if the rate of pumping into it is much larger than its decay rate. In order to quantify this effect, we introduce the parameter $\alpha$ as

$$
\alpha=\frac{\gamma_{4}}{\gamma_{2}+\gamma_{3}}
$$

Thus, for $\Phi=2 n \pi$ and $\alpha \gg 1 \quad(\alpha \ll 1)$ the dark state $\left|\Psi_{23}(\pi)\right\rangle\left(\left|\Psi_{14}(\pi)\right\rangle\right)$ is long lived with respect to all other states and, at steady state, it has a high probability of occupation. This probability increases the more $\alpha$ differs from unity and it approaches 1 for $\alpha \rightarrow \infty(\alpha \rightarrow 0)$, corresponding to the system being trapped in $\left|\Psi_{23}(\pi)\right\rangle\left(\left|\Psi_{14}(\pi)\right\rangle\right)$. We will show that due to this effect population inversion can occur on the transition $|1\rangle \rightarrow|2\rangle,|3\rangle$ for $\alpha \gg 1$ and on $|2\rangle,|3\rangle \rightarrow|4\rangle$ for $\alpha \ll 1$. Such behavior disappears as $\alpha$ approaches 1 , and for $\alpha=1$ and at saturation, the system is equally scattered among all states.

\section{B. Comparison with the double- $\Lambda$ configuration}

In the absence of spontaneous decay, the $\diamond$ configuration is formally identical to the double- $\Lambda$ scheme, extensively studied in the literature [4-6]. Thus, the symmetries induced on the coherent dynamics by the phase are exactly the same [3]. We have discussed, however, that the steady state is critically determined by the concurrence between this symmetry and the relaxation processes. Thus, the introduction of the spontaneous decay leads to critical differences between the two systems. For an easier comparison we are labeling the atomic states of the double- $\Lambda$ system as shown in the inset of Fig. 1. In this scheme the excited states $|1\rangle$ and $|4\rangle$ decay spontaneously into the stable or metastable states $|2\rangle$ and $|3\rangle$. In the $\diamond$ scheme, the excited state $|4\rangle$ decays into the intermediate states $|2\rangle$ and $|3\rangle$, which themselves decay into the ground state $|1\rangle$. A first difference is that in the $\diamond$ scheme the dynamics will be phase sensitive only when the $V$ or the $\Lambda$ scheme (or both) are driven at saturation, while below saturation it will reduce to the well-known $V$ configuration. In the double- $\Lambda$ system, on the other hand, phasesensitive dynamics survives also well below saturation [6].

When looking at the behavior as a function of $\Phi$, the differences are more striking: at $\Phi=(2 n+1) \pi$, for instance, the $\diamond$ scheme is pumped into the subspace $\left\{|1\rangle,\left|\Psi_{23}(0)\right\rangle\right\}$, which is a closed two-level transition, for the coherent drive as well as for the relaxation processes. In the double- $\Lambda$ scheme, instead, the atom can be found in any of the four states due to incoherent coupling [13]. At $\Phi=2 n \pi$, the role of the dark states differs between the two configurations. In the double- $\Lambda$ system CPT occurs in the state $\left|\Psi_{23}(\pi)\right\rangle$, 
which is completely dark and, in the absence of other sources of decay, stable. In this configuration, and for equal decay rates from the excited states, the state $\left|\Psi_{14}(\pi)\right\rangle$ is never accessed. In the $\diamond$ scheme, instead, both dark states are accessed, and (partial) CPT occurs only when their decay rates differ substantially from one another.

\section{STEADY-STATE SOLUTIONS}

In this section we study the steady-state solution of Eq. (9) as a function of the phase $\Phi$. We consider laser frequencies and geometries that fulfill $\Delta k=0$ and $\Delta \omega=0$, so that $\Phi$ does not depend on time and space. In order to obtain simple analytic solutions we consider resonant drives so that $\delta_{j}$ $=0$. Further, we assume that the moduli of the Rabi frequencies are all equal, $g_{i j}=g$, and that the decay rates fulfill the relation $\gamma_{2}=\gamma_{3}=\gamma, \gamma_{42}=\gamma_{43}=\gamma_{4} / 2$. Under these assumptions, the system exhibits symmetry in the clockwise and counterclockwise multiphoton excitation paths, the difference being the phase $\Phi$.

In this limit, we report and discuss the steady-state solutions of the OBE in Eqs. (10)-(19) as a function of the phase $\Phi$ and of the dimensionless parameters $\Omega=g / \gamma$ and $\alpha$ $=\gamma_{4} /(2 \gamma)$, defined in Eq. (23). We remark that in the following the rotated one-photon coherence

$$
\tilde{\rho}_{34}=\rho_{34} e^{\mathrm{i} \Phi}
$$

is reported in the results. This frame allows us to identify the real and imaginary parts of $\rho_{12}, \rho_{13}, \rho_{24}, \tilde{\rho}_{34}$ with the dispersive and absorptive response of the atomic medium to the fields which couple the corresponding transitions [14].

At the end of this section we will discuss experimental situations under which the assumptions given above are justified and discuss our results for generic values of $\delta$, in relation to the assumption of classical motion, and for unequal Rabi frequencies and decay rates.

\section{A. Case $\alpha=1$}

We first consider the case $\alpha=1$. For convenience, we separate the real and imaginary part of the coherences, denoting them with $u_{i j}=\operatorname{Re}\left\{\rho_{i j}\right\}, v_{i j}=\operatorname{Im}\left\{\rho_{i j}\right\}$, respectively (here, $\left.\tilde{u}_{34}=\operatorname{Re}\left\{\tilde{\rho}_{34}\right\}, \tilde{v}_{34}=\operatorname{Im}\left\{\tilde{\rho}_{34}\right\}\right)$. The steady-state solutions of the OBE have the form

$$
\begin{aligned}
\rho_{11}^{(s s)}= & \frac{1}{D}\left[1+\frac{16}{3} \Omega^{2}+\frac{19}{9} \Omega^{4}\left(\sin ^{2} \frac{\Phi}{2}+3\right)\right. \\
& \left.+\frac{4}{9} \Omega^{6}\left(5 \sin ^{2} \frac{\Phi}{2}+3\right)+\frac{2}{9} \Omega^{8} \sin ^{2} \Phi\right], \\
\rho_{22}^{(s s)}= & \rho_{33}^{(s s)}=\frac{\Omega^{2}}{D}\left[1+\frac{1}{3} \Omega^{2}\left(7+3 \sin ^{2} \frac{\Phi}{2}\right)\right. \\
& \left.+\frac{4}{9} \Omega^{4}\left(3+\sin ^{2} \frac{\Phi}{2}\right)+\frac{2}{9} \Omega^{6} \sin ^{2} \Phi\right],
\end{aligned}
$$

$$
\begin{gathered}
\rho_{44}^{(s s)}=\frac{\Omega^{4}}{D} \cos ^{2} \frac{\Phi}{2}\left(1+\frac{4}{3} \Omega^{2}+\frac{8}{9} \Omega^{4} \sin ^{2} \frac{\Phi}{2}\right), \\
u_{12}^{(s s)}=-u_{13}^{(s s)}=\frac{\Omega^{3}}{2 D} \sin \Phi\left[-1+\frac{2}{3} \Omega^{2}+\frac{8}{9} \Omega^{4} \sin ^{2} \frac{\Phi}{2}\right] \\
v_{12}^{(s s)}=v_{13}^{(s s)}=\frac{\Omega}{D}\left[1+\frac{1}{3} \Omega^{2}\left(7+3 \sin ^{2} \frac{\Phi}{2}\right)\right. \\
\left.+\frac{4}{9} \Omega^{4}\left(3+\sin ^{2} \frac{\Phi}{2}\right)+\frac{2}{9} \Omega^{6} \sin ^{2} \Phi\right] \\
u_{24}^{(s s)}=-\tilde{u}_{34}^{(s s)}=\frac{\Omega^{3}}{2 D} \sin \Phi\left(1+2 \Omega^{2}+\frac{8}{9} \Omega^{4} \sin ^{2} \frac{\Phi}{2}\right) \\
v_{24}^{(s s)=} \tilde{v}_{34}^{(s s)}=\frac{\Omega^{3}}{D} \cos ^{2} \frac{\Phi}{2}\left(1+\frac{4}{3} \Omega^{2}+\frac{8}{9} \Omega^{4} \sin ^{2} \frac{\Phi}{2}\right) \\
v_{23}^{(s s)}=-\frac{\Omega^{4}}{D} \sin \Phi\left[1+\frac{2}{3} \Omega^{2} \sin ^{2} \frac{\Phi}{2}\right] \\
u_{14}^{(s s)}=-\frac{\Omega^{2}}{D} \cos ^{2} \frac{\Phi}{2}\left[1+\frac{4}{3} \Omega^{2}+\frac{4}{9} \Omega^{4} \sin ^{2} \frac{\Phi}{2}\right] \\
u_{14}^{(s s)}=\frac{\Omega^{2}}{D}\left[1+\frac{2}{3} \Omega^{2}\left(2+3 \sin ^{2} \frac{\Phi}{2}\right)\right] \\
\sin ^{2} \frac{\Phi}{2} \Phi\left[1+\frac{4}{3} \Omega^{2}+\frac{4}{9} \Omega^{4} \sin ^{2} \frac{\Phi}{2}\right]
\end{gathered}
$$

where

$$
\begin{aligned}
D= & 1+\frac{22}{3} \Omega^{2}+\frac{4}{9} \Omega^{4}\left(7 \sin ^{2} \frac{\Phi}{2}+27\right)+\frac{16}{9} \Omega^{6}\left(\sin ^{2} \frac{\Phi}{2}+3\right) \\
& +\frac{8}{9} \Omega^{8} \sin ^{2} \Phi .
\end{aligned}
$$

The form of the solutions allows us to identify the contributions of the various multiphoton processes to the steady state. For instance, at second order in $\Omega$ (i.e., at second order in $g / \gamma)$ only $\rho_{14}$ depends on the phase while the populations, one-photon coherences and $\rho_{23}$ are independent of $\Phi$, and $\rho_{44}, \rho_{24}, \rho_{34}, u_{12}, u_{13}$, and $v_{23}$ vanish. In fact, this limit corresponds to weak drives, and the relevant processes consist of resonant scattering on the transitions $|1\rangle \rightarrow|2\rangle,|3\rangle$. Thus, at second order in $\Omega$ the system is equivalent to a $V$ configuration driven below saturation.

At higher order, the steady-state solutions are phase dependent. This is evident, e.g., in the excited-state population, which is proportional to $\cos ^{2}(\Phi / 2)$. In particular, at lowest order in $\Omega, \rho_{44} \approx \Omega^{4} \cos ^{2}(\Phi / 2)$. However, as $\Omega$ is increased 

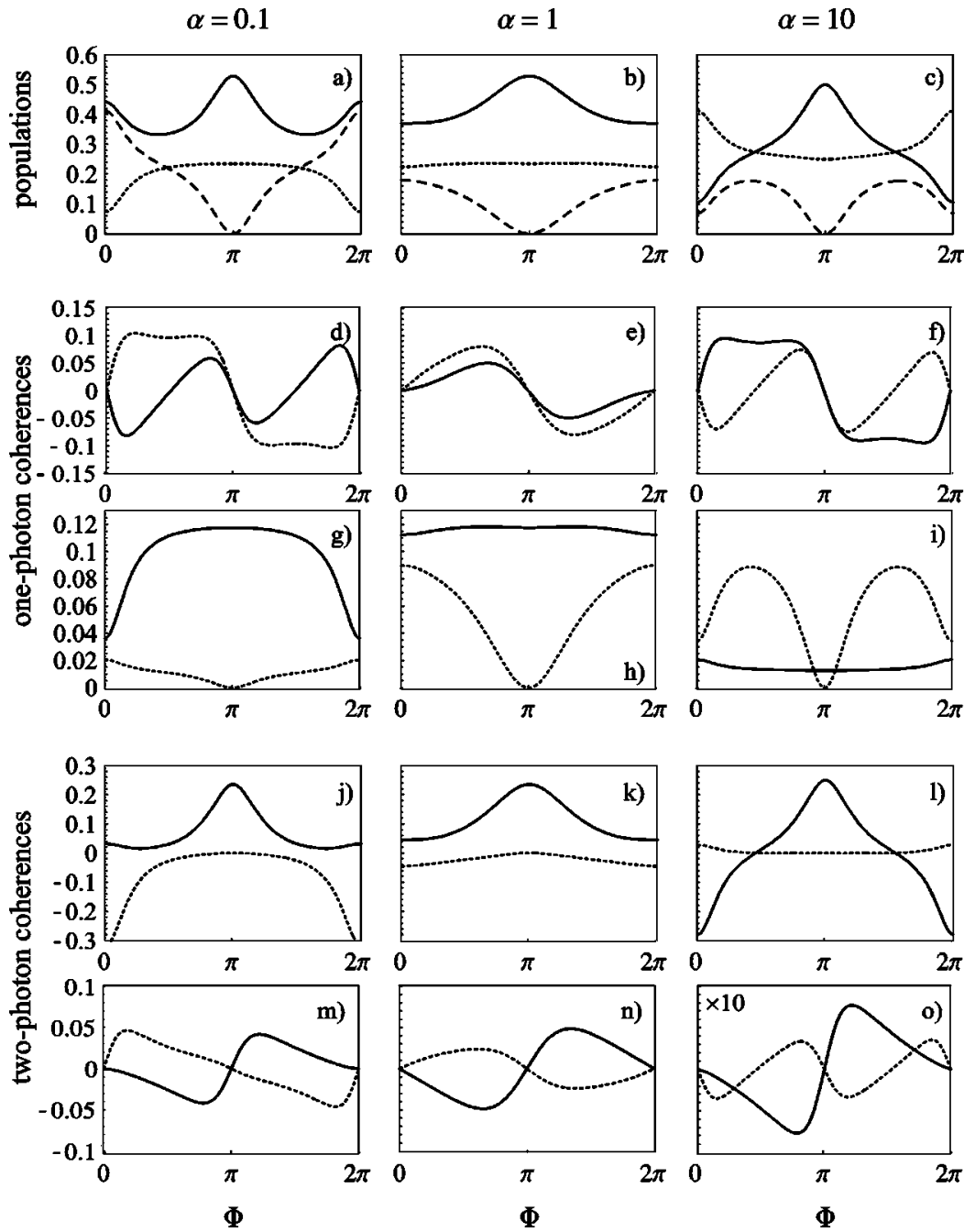

FIG. 3. Steady-state values of populations and coherences as a function of the phase $\Phi$ for resonant drives and different decay rates: $\alpha=0.1$ with $\gamma_{4}=0.2 \gamma$ and $g=2 \gamma$ (left column), $\alpha=1$ with $\gamma_{4}=2 \gamma$ and $g=2 \gamma$ (center column), $\alpha=10$ with $\gamma_{4}=20 \gamma$ and $g=\gamma_{4}$ (right column). Subplots (a), (b), and (c), populations of $\rho_{11}$ (solid), $\rho_{22}=\rho_{33}$ (dotted), and $\rho_{44}$ (dashed line). Subplots (d), (e), and (f), real part of the one-photon coherences; $u_{12}=-u_{13}$ (solid) and $u_{24}=-\tilde{u}_{34}$ (dotted line). Subplots $(\mathrm{g}),(\mathrm{h})$, and (i), imaginary part of the one-photon coherences; $v_{12}=v_{13}$ (solid) and $v_{24}$ $=\tilde{v}_{34}($ dotted line). Subplots $(\mathrm{j}),(\mathrm{k})$, and $(\mathrm{l})$, real part of the two-photon coherences; $u_{23}$ (solid), $u_{14}$ (dotted line). Subplots (m), (n), and (o), imaginary part of the two-photon coherences; $v_{23}$ (solid), $v_{14}$ (dotted line). Note the different scaling factor of the vertical axis in subplot (o).

this modulated dependence of the populations is lost: at leading order in $\Omega$, and for $\Phi \neq(2 n+1) \pi$, all states are equally populated. An exceptional behavior occurs at $\Phi=(2 n$ $+1) \pi$. Here, $\rho_{44}=0$ at all orders, while at leading order $\rho_{11}=2 \rho_{22}=2 \rho_{33}=1 / 2$. In Fig. 3(b) the populations are plotted as a function of the phase for $\Omega=2$ and $\alpha=1$. [For comparison, Figs. 3(a) and 3(c) plot the populations for $\alpha$ $\ll 1$ and $\alpha \gg 1$, respectively; we will discuss these regimes in the following subsection.] For the chosen parameters, $\rho_{11}$, $\rho_{44}$ vary with $\Phi$, while $\rho_{22}, \rho_{33}$ are almost independent of the phase.

Figures 3(e) and 3(h) show the one-photon coherences [Eqs. (28)-(31)] as a function of $\Phi$ for $\Omega=2$ and $\alpha=1$. Their real parts vanish for $\Phi=n \pi$, and one can easily verify from Eqs. (28) - (31) that this occurs at all orders in $\Omega$. This is a feature of resonantly driven two-level systems, and this result is consistent with the analysis of the preceding section. Moreover, for $\Phi=(2 n+1) \pi$ one finds $\rho_{24}=\rho_{34}=0$, which is consistent with the vanishing of $\rho_{44}$. It is worth noting that $u_{12}$ and $u_{13}$ can show additional zeros, as can be seen from their analytic form. These zeros depend on the values of $\Phi$ and $\Omega$, which for $\alpha=1$ satisfy the relation $\sin ^{2}(\Phi / 2)$ $=3\left(3-2 \Omega^{2}\right) / 8 \Omega^{4}$. Thus, they exist only for a certain range of values of the Rabi frequency. Their existence can be interpreted as interference of multiphoton scattering at all orders.

The two-photon coherence $\rho_{14}$ is proportional to $\cos (\Phi / 2)$, in agreement with Eq. (20). The interpretation of the two-photon coherences becomes more transparent by employing the basis of the preceding section. For instance, $\rho_{14}$ can be expressed as

$$
\begin{gathered}
u_{14}=\frac{1}{2}\left[\left\langle\Psi_{14}(0)|\rho| \Psi_{14}(0)\right\rangle-\left\langle\Psi_{14}(\pi)|\rho| \Psi_{14}(\pi)\right\rangle\right] \\
v_{14}=\operatorname{Im}\left\{\left\langle\Psi_{14}(\pi)|\rho| \Psi_{14}(0)\right\rangle\right\} .
\end{gathered}
$$

Analog equations hold for $u_{23}$ and $v_{23}$. Thus, $u_{14}=-1 / 2$ $(+1 / 2)$ corresponds to the system being in the state $\left|\Psi_{14}(\pi)\right\rangle\left(\left|\Psi_{14}(0)\right\rangle\right)$. The imaginary part $v_{14}$ measures the coherence between these two states. We now look at Eqs. (32)-(35) as a function of $\Phi$, which are plotted in Fig. 3(k) and $3(\mathrm{n})$ for $\Omega=2$. The behavior we observe is consistent with the above interpretation in the superposition basis, and with the discussion of the populations and one-photon coherences. At $\Phi=2 n \pi$ the imaginary part $v_{14}\left(v_{23}\right)$ vanishes, supporting the hypothesis of no coherence between $\left|\Psi_{14}(\pi)\right\rangle$ and $\left|\Psi_{14}(0)\right\rangle\left(\left|\Psi_{23}(\pi)\right\rangle\right.$ and $\left.\left|\Psi_{23}(0)\right\rangle\right)$. More- 
Populations

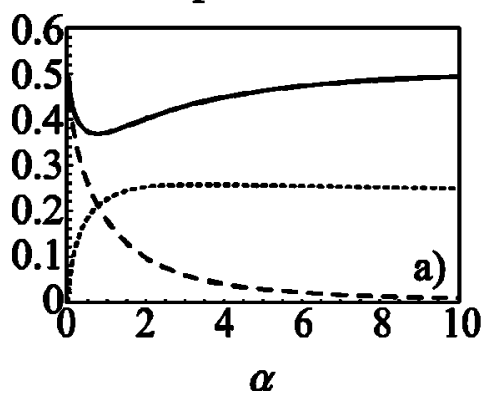

One-photon coherences

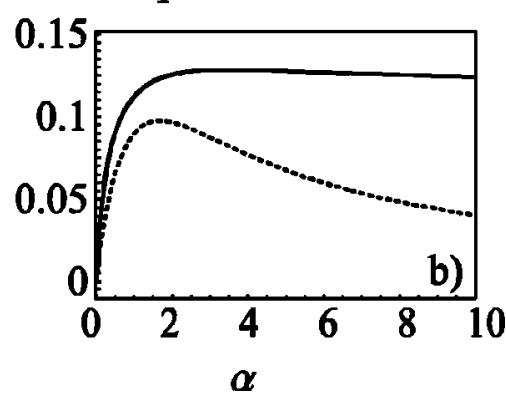

\section{Two-photon coherences}

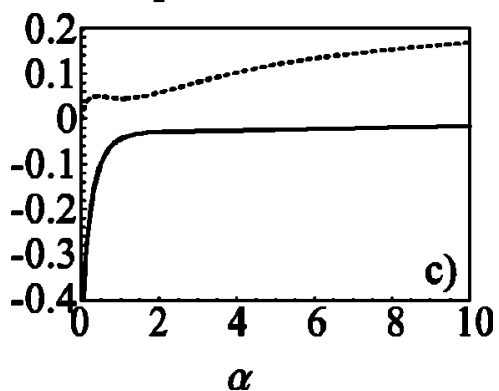

FIG. 4. Level populations and associated coherences for $\Phi=2 n \pi$ as a function of the balance of the decay rates $\alpha$ for $g=2 \gamma$ (where $\gamma$ is kept constant). (a) Populations: $\rho_{11}$ (solid line), $\rho_{22}=\rho_{33}$ (dotted line), $\rho_{44}$ (dashed line). (b) One-photon coherences: $v_{12}=v_{13}$ (solid line), $v_{24}=\tilde{v}_{34}$ (dotted line). (c) Two-photon coherences: $u_{23}$ (solid line), $u_{14}$ (dotted line). All other coherences vanish.

over, $u_{14}=-u_{23}<0$, which implies, after a straightforward calculation, that the probability to find the system in the dark states is $1 / 2$. Thus, it is not proper to speak of CPT for these parameters.

At leading order in $\Omega$ the coherences vanish for $\Phi$ $=2 n \pi$, in agreement with the expectation that at saturation the system is equally distributed between all states. At $\Phi$ $=(2 n+1) \pi$ one finds $\rho_{14}=v_{23}=0$ while $u_{23}$ is positive and exhibits a local maximum. This is consistent with the picture of two-level dynamics between $|1\rangle$ and $\left|\Psi_{23}(0)\right\rangle$.

So far we have discussed the case $\alpha=1$, when the relaxation rates of the two-photon coherences are the same. We have seen that the features of the phase-induced dynamics are always recognizable in the coherences. However, at steady state the atom is not localized in a particular atomic level or coherent superposition of atomic levels. In general, the dependence of the populations on the phase is washed out for increasing Rabi frequencies, except for the vanishing of $\rho_{44}$ at $\Phi=(2 n+1) \pi$. This is understood by considering that the steady state is given by the concurrence of the coherent drive, which has a phase-dependent symmetry, and the relaxation processes with a fixed structure of the coupling between the atomic states. For any value of $\Phi \neq(2 n+1) \pi$, the two effects compete, and at saturation the atomic states are equally populated. On the contrary, for $\Phi=(2 n+1) \pi$, an eigenspace of the coherent scattering processes exists and is preserved by the action of the incoherent processes. Consequently, at any value of $\Omega$ and $\alpha$ the occupation of the state $|4\rangle$ vanishes.

\section{B. Case $\alpha \neq 1$}

Figures 3(a)-3(o) plot the steady-state solutions of the OBE for $\alpha=0.1,1,10$. Comparing the curves, we see some general features in the behavior at different $\alpha$. For instance, the population of the state $|4\rangle$ is always zero for $\Phi=(2 n$ $+1) \pi$. This value of the phase is also a pole of the coherences $\rho_{24}, \rho_{34}, \rho_{14}$, and of the real parts $u_{12}, u_{23}$. Here, the population of the state $|1\rangle$ and the real part of the two-photon coherence $u_{23}$ exhibit a local maximum. These results are all consistent with the picture of two-level dynamics between $|1\rangle$ and $\left|\Psi_{23}(0)\right\rangle$, as discussed in the preceding section. The steady-state values have a very transparent form for $\Phi$ $=(2 n+1) \pi$, and read

$$
\begin{gathered}
\rho_{11}^{s s}=\frac{1+2 \Omega^{2}}{1+4 \Omega^{2}}, \\
\rho_{22}^{s s}=\rho_{33}^{s s}=\frac{\Omega^{2}}{1+4 \Omega^{2}}, \\
\rho_{44}^{s s}=0, \\
v_{12}^{s s}=v_{13}^{s s}=\frac{\Omega}{1+4 \Omega^{2}}, \\
u_{23}^{s s}=\frac{\Omega^{2}}{1+4 \Omega^{2}}, \\
u_{12}^{s s}=u_{13}^{s s}=\rho_{24}^{s s}=\rho_{34}^{s s}=v_{23}^{s s}=\rho_{14}^{s s}=0,
\end{gathered}
$$

which have been evaluated for $\Omega=g / \gamma$ and an arbitrary value of $\alpha=\gamma_{4} / 2 \gamma$. In these solutions, the parameter $\gamma_{4}$ does not appear, showing once again that the level $|4\rangle$ does not affect the steady-state dynamics for this value of the phase.

A striking difference among the three regimes appears at values of the phase close to $\Phi=2 n \pi$. Here, we find population inversion on the transitions $|1\rangle \rightarrow|2\rangle,|3\rangle$ for $\alpha=10$, $(|2\rangle,|3\rangle \rightarrow|4\rangle$ for $\alpha=0.1)$, while the real part of the twophoton coherence $u_{23}\left(u_{14}\right)$ approaches the value $-1 / 2$. At this value of $\Phi$, we write the steady-state solutions as a function of $\gamma, \alpha=\gamma_{4} / 2 \gamma$ and $\Omega=g / \gamma$,

$$
\begin{gathered}
\rho_{11}^{s s}=\frac{1}{D}\left[\alpha^{2}(1+2 \alpha)+\Omega^{2} \alpha\left(3+5 \alpha+4 \alpha^{2}\right)+\Omega^{4}(1+2 \alpha)\right], \\
\rho_{22}^{s s}=\rho_{33}^{s s}=\frac{\alpha \Omega^{2}}{D}\left[\alpha(1+2 \alpha)+\Omega^{2}(\alpha+2)\right] \\
\rho_{44}^{s s}=\frac{\Omega^{4}}{D}(1+2 \alpha), \\
v_{12}^{s s}=v_{13}^{s s}=\frac{\alpha \Omega}{D}\left[\alpha(1+2 \alpha)+\Omega^{2}(2+\alpha)\right],
\end{gathered}
$$




$$
\begin{gathered}
v_{24}^{s s}=\tilde{v}_{34}^{s s}=\frac{\alpha \Omega^{3}}{D}(1+2 \alpha), \\
u_{23}^{s s}=\frac{\alpha \Omega^{2}}{D}\left[\alpha(1+2 \alpha)+\Omega^{2}(1-\alpha)\right], \\
u_{14}^{s s}=-\frac{\Omega^{2}}{D}\left[\alpha(1+2 \alpha)+\Omega^{2}(1-\alpha)\right], \\
u_{12}^{s s}=u_{13}^{s s}=v_{14}^{s s}=v_{23}^{s s}=u_{24}^{s s}=\tilde{u}_{34}^{s s}=0,
\end{gathered}
$$

where

$$
D=\alpha^{2}(1+2 \alpha)+\Omega^{2} \alpha\left(3+7 \alpha+8 \alpha^{2}\right)+2 \Omega^{4}\left(1+4 \alpha+\alpha^{2}\right) .
$$

These results are plotted as a function of $\alpha$ in Fig. 4, by keeping $\gamma$ and $\Omega$ as fixed parameters. Here, we see that for $\alpha \ll 1$ the system is localized in the atomic states $|1\rangle,|4\rangle$, and the coherence $u_{14}$ has a maximum absolute value. In particular, for $\alpha \rightarrow 0$ the populations of the states $|2\rangle,|3\rangle$ vanish together with the imaginary part of all coherences. In this case the atom is in the dark state $\left|\Psi_{14}(\pi)\right\rangle$, which is stable, and CPT occurs. Such localization persists for small values of $\alpha$, although the populations of the intermediate states, and the incoherent scattering processes, increase as $\alpha$ approaches 1. It is interesting that for these (small) values of $\alpha$ the system exhibits population inversion on the transitions $|2\rangle,|3\rangle$ $\rightarrow|4\rangle$. Analogously, it can be verified that, fixed $\gamma_{4}$ and $g$, for $\gamma \rightarrow 0$ the system is trapped in the dark state $\left|\Psi_{23}(\pi)\right\rangle$ : CPT occurs in this coherence, and this implies population inversion on the transition $|1\rangle \rightarrow|2\rangle,|3\rangle$. Note that the localization in an atomic superposition persists in the neighborhood of the value of the phase $\Phi=2 n \pi$, as it is visible in Figs. 3(a) and 3(c). For instance, for $\alpha \gg 1$ the population inversion occurs on the transition $|2\rangle,|3\rangle \rightarrow|1\rangle$ on an interval of values $\left[2 n \pi-\Phi_{0}, 2 n \pi+\Phi_{0}\right]$. The phase $\Phi_{0}$ satisfies the relation $\rho_{22}\left(\Phi_{0}\right), \rho_{33}\left(\Phi_{0}\right)=\rho_{11}\left(\Phi_{0}\right)$, and in general $\Phi_{0}$ can be said to separate two regimes, where the dynamics associated with the symmetry at phase $2 n \pi$ or with $\Phi$ $=(2 n+1) \pi$ prevails.

It is interesting to note that for $\Phi=2 n \pi$ the populations and, in particular, the decay-dependent population inversion show trends typical of a three-level cascade system while for $\Phi=(2 n+1) \pi$ the system is effectively reduced to a $V$ configuration because of $\rho_{44}=0$.

Finally, we emphasize the additional poles of the onephoton coherences which we have identified in the analytical solutions for $\alpha=1$. We have interpreted their origin as due to photon scattering at all orders. We remark that they appear in $u_{12}$ and $u_{13}$ for $\alpha=0.1$, see Fig. 3(d), and in $u_{24}$ and $\tilde{u}_{34}$ for $\alpha=10$, see Fig. 3(f).

\section{Discussion}

The analysis of this section is restricted to the choice of parameters $\delta_{j}=0$, which corresponds here to values of $p_{z}$ $\approx 0$. This describes the behavior of a gas after Doppler cooling, at a thermal energy of $\kappa_{B} T \sim \hbar \Gamma / 2$, with $\Gamma$ $=\min \left(\gamma, \gamma_{4}\right)$. Provided that the linewidth $\Gamma$ is much larger than the recoil frequencies, $\Gamma \gg \hbar k_{i j}^{2} / 2 m$, the presented results describe sensibly the atomic response to the drive.

When the medium is Doppler broadened, i.e., for $\kappa_{B} T$ $>\hbar \Gamma / 2$ (still keeping the constraint on the recoil energies), many features discussed for the case $p_{z}=0$ survive and will appear in the signal measured over the ensemble, provided that $\delta_{2}=\delta_{3}=\delta \delta_{4}=0$, so that two-photon transitions are Doppler-free. This situation can be realized for degenerate intermediate-state energies $\left(\omega_{2}=\omega_{3}\right)$, resonant drives $\left(\omega_{12}\right.$ $\left.=\omega_{13}=\omega_{2}-\omega_{1}, \omega_{24}=\omega_{34}=\omega_{4}-\omega_{2}\right)$, and laser geometries such that the wave vectors fulfill the relation $k_{12}=k_{13} \sim$ $-k_{24}=-k_{34}=k$. In this way $\Phi$ does not depend on time and space $(\Delta \omega=0, \Delta k=0)$, and $\delta$ is given by $k p_{z} / m$. Also in this regime we find that for $\Phi=(2 n+1) \pi$ the population of 14) vanishes independently of $\delta$, together with the coherences $\rho_{24}, \rho_{34}$, and $\rho_{14}$. For $\Phi=2 n \pi$ and $\alpha$ sufficiently different from unity, population inversion can be observed, provided the atomic transitions are saturated [15].

Finally, we remark that only a part of these considerations can be applicable to "asymmetric" configurations, i.e., for values of the Rabi frequencies, eigenenergies, decay rates, etc., which change the structure of the Hamiltonian and relaxation processes, introducing thus either different weights to the interfering excitation paths, and/or additional relative phases, and/or different resonances. Here, the dependence of the steady state on the phase $\Phi$ cannot often be simply singled out. The dynamics is a complex combination of all parameters, and exhibits an extremely rich variety of phenomena, which will be subject to future investigations.

\section{CONCLUSION}

We have studied the dynamics of a four-level system interacting with lasers in a configuration which we have labeled the $\diamond$ scheme because of its geometry. This scheme has a closed-loop excitation structure $[3,4]$, i.e., any transition amplitude between two given states is the sum of two contributions, corresponding to two excitation paths, which may interfere. The dynamics is determined by a large number of parameters. Here, we have considered that both paths have the same weight, while they differ by a relative phase $\Phi$. We have discussed the origin of $\Phi$, and investigated the steady state of the interacting system as a function of $\Phi$, in the regime where the steady-state solution exists.

For the chosen parameters, the steady-state solution is phase sensitive. This is particularly evident in the coherences, whereas, in general, the phase dependence of the population is particularly enhanced for certain ranges of values of the relaxation rates. In particular, when the lifetimes of the intermediate states are considerably different from that of the upper state, the system can exhibit population inversion for some values of the phase around $\Phi=2 n \pi$. We have interpreted and discussed this result in terms of coherent population trapping. Nevertheless, in all regimes here considered the population of the upper state vanishes for $\Phi$ $=(2 n+1) \pi$. We have explained these behaviors using a convenient basis, showing that the dynamics is given by the concurrence of the Hamiltonian evolution, which is phase 
sensitive, with the structure and nonunitarity of the relaxation processes. In particular, for $\Phi=(2 n+1) \pi$ the steady state of the system corresponds to the steady state of a (closed) two-level transition.

The phase dependence of the Hamiltonian evolution in closed-loop schemes shares many analogies with an atom interferometer [3]. The phase dependence survives also at steady state $[4,5]$, and the response of the system could be used as a device for measuring the relative phase between laser fields. For instance, in the $\diamond$ system the phase could be measured through the population of the upper state. In fact, for sufficiently weak fields, the functional behavior of this population is well approximated by $\cos ^{2} \Phi / 2$, and the fluorescence signal from the upper state shows the features of an interference pattern which is sensitive to $\Phi$.

This study may be useful in the spectroscopy of alkaliearth atoms, currently investigated in experiments aiming at optical frequency standards [10] or at Bose-Einstein condensation by all-optical means [11]. Further, efficient laser- cooling schemes for these kind of atoms could be developed, by exploiting the phase properties due to the atomic motion in proper laser geometries [16].

Finally, the $\diamond$ scheme exemplifies a system where nonlinear optics with resonant atoms can be realized. Here, the phase is a control parameter capable to change the response of the medium to the drive $[5-8,17,18]$. This will be the object of future investigations.

\section{ACKNOWLEDGMENTS}

This work has been motivated by a problem posed by Robin Kaiser and Ennio Arimondo who are kindly acknowledged. We also thank S.M. Barnett, W. Lange, M. Scully, E. Solano, H. Walther, and in particular P. Lambropoulos for useful discussions and comments. We acknowledge support from the TMR-networks QSTRUCT and QUANTIM, EPSRC (Grant No. GR/R04096) and the Leverhulme Trust. G.L.O. acknowledges support from SGI.
[1] Allen and Eberly, Optical Resonance and Two-level Atom (Wiley, New York, 1975).

[2] S.E. Harris, Phys. Today 50(7), 36 (1997); E. Arimondo, Prog. Opt. 35, 259 (1996).

[3] S.J. Buckle, S.M. Barnett, P.L. Knight, M.A. Lauder, and D.T. Pegg, Opt. Acta 33, 1129 (1986).

[4] D.V. Kosachiov, B.G. Matisov, and Y.V. Rozhdestvensky, J. Phys. B 25, 2473 (1992).

[5] E.A. Korsunsky and D.V. Kosachiov, Phys. Rev. A 60, 4996 (1999).

[6] M.D. Lukin, P.R. Hemmer, and M.O. Scully, Adv. At., Mol., Opt. Phys. 42, 347 (2000).

[7] W. Maichen, F. Renzoni, I. Mazets, E. Korsunsky, and L. Windholz, Phys. Rev. A 53, 3444 (1996).

[8] A.J. Merriam, S.J. Sharpe, M. Shverdin, D. Manuszk, G.Y. Yin, and S.E. Harris, Phys. Rev. Lett. 84, 5308 (2000).

[9] G. Grynberg and P.R. Berman, Phys. Rev. A 41, 2677 (1990).

[10] T. Binnewies, G. Wilpers, U. Sterr, F. Riehle, J. Helmcke, T.E. Mehlstäubler, E.M. Rasel, and W. Ertmer, Phys. Rev. Lett. 87, 123002 (2001); E.A. Curtis, C.W. Oates, and L. Hollberg, Phys. Rev. A 64, 031403(R) (2001).
[11] H. Katori, T. Ido, Y. Isoya, and M. Kuwata-Gonokami, Phys. Rev. Lett. 82, 1116 (1999).

[12] Our analysis is restricted to the limit in which the internal relaxation is much faster than the rate with which the atomic center-of-mass coordinates evolve on a macroscopic scale. On this short time scale we evaluate the internal steady state, treating $z, p_{z}$ as fixed parameters.

[13] Note that for $\Phi=(2 n+1) \pi$ population inversion has been predicted in the double- $\Lambda$ configuration for different decay rates from the excited states [4].

[14] M.O. Scully and M.S. Zubairy, Quantum Optics (Cambridge University Press, Cambridge, 1997).

[15] S. Franke-Arnold et al. (unpublished).

[16] D.V. Kosachiov, B.G. Matisov, and Y.V. Rozhdestvensky, Europhys. Lett. 22, 11 (1993); T. Pellizzari and H. Ritsch, ibid. 31, 133 (1995).

[17] E.A. Korsunsky, N. Leinfellner, A. Huss, S. Baluschev, and L. Windholz, Phys. Rev. A 59, 2302 (1999).

[18] C. Affolderbach, S. Knappe, R. Wynands, A.V. Taichenachev, and V.I. Yudin, Phys. Rev. A 65, 043810 (2002). 